\title{
THE AXIOM OF CHOICE FOR FINITE SETS
}

\author{
R. L. BLAIR AND M. L. TOMBER
}

If $\mathcal{F}$ is a family of nonempty sets, then by a choice function on $\mathcal{F}$ we mean a function $f$ on $\mathcal{F}$ such that $f(S) \in S$ for every $S \in \mathcal{F}$. The axiom of choice for finite sets may be formulated as follows:

(ACF) If $\mathcal{F}$ is any family of nonempty finite sets, then there exists a choice function on $\mathcal{F}$.

We shall denote the axiom of choice (for families of arbitrary nonempty sets) by (AC). It is easy to see that the ordering principle (which asserts that every set can be totally ordered) implies (ACF). ${ }^{1}$ On the other hand, Mostowski [4] has shown that (relative to a suitable system of axiomatic set theory) the ordering principle is actually weaker than (AC) so that (ACF), while it is surely a consequence of $(A C)$, is not equivalent to $(A C)$ [4, Korollar II, p. 250].

The object of the present note is to obtain equivalent formulations of (ACF) of the Zorn's lemma type. In fact, in $\$ 1$ we show that $(\mathrm{ACF})$ is equivalent to both (ZLF1) and (ZLF2) below; the latter are obtained by restricting two familiar "maximal element" forms of Zorn's lemma to a special class of partially ordered sets, namely, to those "with finitary covers" (see the definition below). It is noteworthy, however, that not every form of Zorn's lemma, when so restricted, is equivalent to (ACF). For example, in $\$ 2$ we observe that if the statement of either (ZLF1) or (ZLF2) is modified merely by replacing the hypothesis of a least upper bound by that of an upper bound, then the resulting formulation is equivalent, not to (ACF), but to (AC) itself. And furthermore, when one formulates the natural "maximal chain" analogue of (ZLF1) and (ZLF2), one finds that the result is again equivalent not to (ACF) but to the full axiom of choice (AC).

The first named author is pleased to record his indebtedness to Professor Herman Rubin for a number of instructive conversations on the subject of this note.

1. Zorn's lemma formulations of (ACF). Let $P$ be a partially ordered set. For $x, y \in P$, define $x \sim y$ in case either (i) $x=y$ or (ii) $x$

Received by the editors May 22, 1959.

1 Tarski $[9$, p. 82] attributes this observation to Kuratowski; for certain related remarks, see $[2 ; 3]$, and $[10]$. For a study of certain variants of (ACF) (in which cardinality restrictions are placed on the sets in $F$ ), see Mostowski [5], Szmielew [8], and Sierpinski [7]. 
and $y$ have no common upper bound. It is clear that the relation $\sim$ is both reflexive and symmetric.

If $x, y \in P$, then $x$ covers $y$ in case $x>y$ and $x>z>y$ for no $z \in P$. If $x \in P$, then we shall denote by $C(x)$ the set of all elements of $P$ that cover $x$.

It will be convenient to introduce the following definition:

Definition. We say that $P$ is a partially ordered set with finitary covers in case for each nonmaximal element $x \in P$ the following conditions hold:

(F1) $C(x)$ is not empty.

(F2) If $a \in C(x)$, then there exists a positive integer $n(a)$ such that any sequence $a=a_{1} \sim a_{2} \sim a_{3} \sim \ldots$ in $C(x)$ contains at most $n(a)$ distinct terms.

(F3) If $X$ is a nonempty subset of $C(x)$ and if every pair of elements of $X$ have a common upper bound, then $X$ has a least upper bound.

We can now formulate the following restricted forms of Zorn's lemma:

(ZLF1) If $P$ is a partially ordered set with finitary covers, and if every well-ordered subset of $P$ has a least upper bound, then $P$ contains a maximal element.

(ZLF2) If $P$ is a partially ordered set with finitary covers, and if every totally ordered subset of $P$ has a least upper bound, then $P$ contains a maximal element.

THEOREM 1. The axiom of choice for finite sets is equivalent to both (ZLF1) and (ZLF2).

A key step in the proof depends upon the following lemma due to Bourbaki:2

Lemma (Bourbaki). If $P$ is a partially ordered set such that every well-ordered subset of $P$ has a least upper bound, and if $\phi$ is a mapping of $P$ into itself such that $x \leqq \phi(x)$ for every $x \in P$, then $\phi(x)=x$ for some $x \in P$.

Proof of Theorem 1 . We shall verify the implications (ACF) $\rightarrow($ ZLF1 $) \rightarrow($ ZLF2 $) \rightarrow(\mathrm{ACF})$. Assume first that $(\mathrm{ACF})$ holds and consider a partially ordered set $P$ with finitary covers. We suppose that every well-ordered subset of $P$ has a least upper bound but that

2 The lemma is an immediate consequence of Theorème 1 and Corollaires 1 and 2 of [1] (cf. N. Bourbaki, Éléments de mathématique, Vol. I; Théorie des ensembles, Chapter III, Paris, 1956, pp. 48-49); no form of the axiom of choice is used in its proof. 
no element of $P$ is maximal. Consider first any element $x \in P$; by (F1) the set $C(x)$ is not empty. We define the relation $R_{x}$ on $C(x)$ as follows: For $a, b \in C(x), a R_{x} b$ in case for some positive integer $m$ there exist elements $x_{i} \in C(x)(i=1, \cdots, m)$ such that

$$
a \sim x_{1} \sim x_{2} \sim \cdots \sim x_{m} \sim b .
$$

Then $R_{x}$ is an equivalence relation on $C(x)$, and, for $a \in C(x)$, we denote by $R_{x}[a]$ the equivalence class of $C(x)$ with representative $a$. It follows from (F2) that $R_{x}[a]$ is finite for each $a \in C(x)$. Then

$$
\mathcal{F}=\bigcup\left\{\left\{R_{x}[a]: a \in C(x)\right\}: x \in P\right\}
$$

is a family of nonempty finite sets so that, by (ACF), there exists a choice function $f$ on $\mathcal{F}$. For each $x \in P$, set

$$
E(x)=\left\{f\left(R_{x}[a]\right): a \in C(x)\right\} .
$$

It is clear that every pair of elements of $E(x)$ have a common upper bound in $P$, and hence, by (F3), $E(x)$ has a least upper bound $\phi(x) \in P$. But then the mapping $x \rightarrow \phi(x)$ has the property that $x<\phi(x)$ for every $x \in P$, which is contrary to the lemma. We conclude that $P$ must have a maximal element and that (ACF) implies (ZLF1).

Since the implication (ZLF1) $\rightarrow(Z L F 2)$ is trivial, let us assume (ZLF2) and consider a family $\mathfrak{F}$ of nonempty finite sets. Denote by $P$ the set of all $f$ such that $f$ is a choice function on some subfamily of $\mathcal{F}$. Partially order $P$ by defining $f \leqq g$ in case $g$ is an extension of $f$, and, for $f \in P$, denote by $D(f)$ the domain of $f$. It is easy to see that every totally ordered subset of $P$ has a least upper bound. Moreover, it is clear that a function $f \in P$ is a choice function on $\mathcal{F}$ if (and only if) $f$ is maximal in $P$. To verify (ACF) it will therefore suffice to show that $P$ satisfies conditions (F1)-(F3).

We consider a nonmaximal element $f \in P$. Then $S \notin D(f)$ for some $S \in \mathcal{F}$. Choosing an element $x \in S$, define $g(S)=x$ and $g(T)=f(T)$ for $T \in \mathscr{D}(f)$. Then $g \in C(f)$ and condition (F1) is satisfied.

Next, it is easy to see that $g \in C(f)$ if and only if $D(g)=D(f) \cup\left\{S_{g}\right\}$ for some (unique) set $S_{\theta} \in \mathcal{F}-\mathscr{D}(f)$, from which it follows that, for $g, h \in C(f), g \sim h$ if and only if $S_{g}=S_{h}$. Thus $\sim$ is an equivalence relation on $C(f)$. We claim that each -equivalence class $Q$ of $C(f)$ is finite. To see this, let $g \in Q$ and suppose that $S_{h} \neq S_{o}$ for some $h \in Q$. If we define $k\left(S_{g}\right)=g\left(S_{g}\right), k\left(S_{h}\right)=h\left(S_{h}\right)$, and $k(T)=f(T)$ for $T \in \mathscr{D}(f)$, then $k \in P$ and $g \leqq k, h \leqq k$, a contradiction. Hence $S_{h}=S_{o}$ for every $h \in Q$. But $S_{o}$ is finite, from which we conclude that $Q$ is finite. It is now clear that $P$ satisfies condition (F2).

Finally, let $X$ be a nonempty subset of $C(f)$ such that every pair 
of elements of $X$ have a common upper bound. Then $S_{o} \neq S_{h}$ for every pair of distinct functions $g, h \in X$. We can therefore define a function $k \in P$ as follows: $k(T)=f(T)$ for $T \in D(f)$, and, for each $g \in X, k\left(S_{\theta}\right)=g\left(S_{\theta}\right)$. It is then clear that $k$ is the least upper bound of $X$ and condition (F3) is satisfied. The proof is now complete.

2. Some equivalent formulations of (AC). Let us denote by $\left(Z L F 1^{*}\right)$ and $(Z L F 2 *)$ the modifications of (ZLF1) and (ZLF2), respectively, obtained by replacing, in their statements, "least upper bound" by "upper bound."

The following observation, originally devised for a slightly different purpose, was communicated to us by Herman Rubin: Let $P$ be any partially ordered set, let $N$ be the set of all positive integers with its usual order, and partially order the cartesian product $Q=P \times N$ lexicographically. Then (as one easily sees) the following statements are equivalent: (i) Every totally ordered subset $K$ of $P$ has an upper bound $x \notin K$. (ii) Every totally ordered subset of $Q$ has an upper bound.

Now for each element $(x, n) \in Q, C((x, n))$ consists precisely of the single element $(x, n+1)$. Hence $Q$ trivially satisfies conditions (F1)(F3). Since $Q$ obviously has no maximal element, both (ZLF1*) and (ZLF2*) imply the existence in $P$ of a totally ordered subset $K$ having no upper bound $x \notin K$, and from this we infer (AC). We therefore have the following result:

TheOREM 2. The axiom of choice is equivalent to both (ZLF1*) and (ZLF2*).

It is a familiar fact that $(\mathrm{AC})$ is equivalent to the "maximal chain theorem": If $P$ is a partially ordered set, then every chain (= totally ordered set) in $P$ is contained in a maximal chain. In view of Theorem 1 , it is therefore natural to consider the following restricted form of the maximal chain theorem:

(MCTF) If $P$ is a partially ordered set with finitary covers, then every chain in $P$ is contained in a maximal chain.

TheOrem 3. The axiom of choice is equivalent to (MCTF).

Proof. $^{3}$ Since (AC) obviously implies (MCTF), let us assume (MCTF) and consider an arbitrary partially ordered set $P$ and a chain $C \subseteq P$. Denote by $\mathcal{e}$ the family of all chains in $P$ and partially order $P=\mathcal{e} \cup\{P\}$ by inclusion. It is clear that $P$ satisfies condition $(\mathrm{F} 1)$,

${ }^{3}$ This is an adaptation of an argument due to Scott [6]. We are indebted to Professor Rubin for calling our attention to Scott's argument and to its applicability in the present context. 
and, since $P$ is a complete lattice, $P$ also satisfies (F2) and (F3). Therefore $P$ contains a maximal chain $\mathcal{K}$ such that $C \in \mathcal{K}$, and hence $K=\mathrm{U}\{A: A \in \mathfrak{K} \cap \mathcal{e}\}$ is a maximal chain in $P$ containing $C$. We conclude that (MCTF) implies (AC).

\section{REFERENCES}

1. N. Bourbaki, Sur le thêorème de Zorn, Arch. Math. vol. 2 (1949-1950) pp. 434437.

2. L. A. Henkin, Metamathematical theorems equivalent to the prime ideal theorems for Boolean algebras, Bull. Amer. Math. Soc. Abstract 60-4-553.

3. J. Łos and C. Ryll-Nardzewski, Effectiveness of the representation theory for Boolean algebras, Fund. Math. vol. 41 (1954) pp. 49-56.

4. A. Mostowski, Über die Unabhängigkeit des Wohlordnungssatzes vom Ordnungsprinzip, Fund. Math. vol. 32 (1939) pp. 201-252.

5. - Axiom of choice for finite sets, Fund. Math. vol. 33 (1945) pp. 137-168.

6. D. Scott, The theorem on maximal ideals in lattices and the axiom of choice, Bull. Amer. Math. Soc. Abstract 60-1-172.

7. W. Sierpinski, L'axiome du choix pour les ensembles finis, Matematiche, Catania vol. 10 (1955) pp. 92-99.

8. Wanda Szmielew, On choices from finite sets, Fund. Math. vol. 34 (1947) pp. 75-80.

9. A. Tarski, Sur les ensembles finis, Fund. Math. vol. 6 (1924) pp. 45-95.

10. - Prime ideal theorems for Boolean algebras and the axiom of choice, Bull. Amer. Math. Soc. Abstract 60-4-562.

UNIVERSITY OF OREgON AND

Michigan State University 